\title{
THE ANALYTICAL TREATMENT OF RESISTIVE WAKE IN ROUND PIPE
}

\author{
M.Ivanyan ${ }^{\dagger}$, V. Tsakanov, YerPhI, Yerevan, Armenia
}

\section{Abstract}

An analytical form of longitudinal and transverse resistive wake potentials of point-like charge, moving parallel to the axe of round pipe with the walls of finite and frequency independent conductivity is obtained. The short range presentation of wake potentials by help of uniformly converged series is received. The series expansion for Gaussian bunch is obtained as well.

The analytical form of resonant term of wake potential and simple integral form of the non-resonance one are given for case of frequency dependent conductivity.

\section{INTRODUCTION}

An analytical form of impedance of infinite round pipe with the resistive walls material is obtained by Chao [1], and was simplified by Bane [2] by dropping out very low and very high frequency terms:

$$
Z(\kappa)=2 /\left(c b s_{0}[\lambda / \kappa-i \kappa \alpha / 2]\right),
$$

where: $b$ is a pipe radius, $s_{0}=\left(c b^{2} / 2 \pi \sigma\right)^{1 / 3}$ is a characteristic distance of the pipe, $c$ is a light velocity, $\sigma$ is a walls material conductivity, $\alpha=b / s_{0}^{2}$ and $\kappa=k s_{0}$ is a dimensionless wavenumber. The parameter $\lambda$ in (1) for arbitrary frequency dependence of conductivity is presented as $\lambda^{2}=4 \pi \sigma i \kappa / c s_{0}$. The wake potential is given by

$$
W_{z}(s)=\left(2 \pi s_{0}\right)^{-1} c \int_{-\infty}^{\infty} Z(\kappa) e^{-i \kappa s / s_{0}} d \kappa
$$

The results of integration of (2) in the complex plane for the frequency independent ( $\mathrm{dc}$ - 'direct current') conductivity is the sum of resonance (given by help of elementary functions) and non-resonance (given in the simple integral form) terms [2]. The last term is performed here to the analytical form.

For the frequency dependent (ac - 'alternating current') conductivity case on the base of numerical calculation method, given in [2], we are brought the analytical form for the resonance term and the simple calculated integral for the non-resonance one.

\section{DC CONDUCTIVITY}

\subsection{Wake Function}

The longitudinal and transversal wake functions in the dc case may be presented in form of expansion by the

ivanian@jerewan1.yerphi.am longitudinal $\left(w_{z . n}\right)$ and transversal $\left(\vec{w}_{r . n}\right)$ multipole moments [2]:

$$
w_{z, r}(s, \vec{r})=\sum_{n=0}^{\infty} w_{z, n .}(s, \vec{r}), w_{z, r}(s, \vec{r})=\sum_{n=1}^{\infty} w_{r, n .}(s, \vec{r}),
$$

where

$$
\begin{aligned}
& w_{z n}(s, \vec{r})=-\frac{4}{b^{2}}\left(\frac{r r_{1}}{b^{2}}\right)^{n} \delta_{n} \gamma_{n} f_{z}\left(u_{n}\right) \cos n\left(\phi-\phi_{1}\right) \\
& \vec{w}_{r n}(s, \vec{r})=\frac{4 s_{0} n}{b^{3} r}\left(\frac{r r_{1}}{b^{2}}\right)^{n} \gamma_{n}^{1 / 3} f_{r}\left(u_{n}\right) \times \\
& \left(\cos n\left(\phi-\phi_{1}\right) \vec{e}_{r}-\sin n\left(\phi-\phi_{1}\right) \vec{e}_{\phi}\right)
\end{aligned}
$$

with

$$
\begin{aligned}
& f_{z}(u)=\frac{4}{3} e^{-u} \cos (\sqrt{3} u)-\frac{4 \sqrt{2}}{\pi} I_{z}(u) \\
& f_{z}(u)=\frac{2}{3} e^{-u}(\sqrt{3} \sin (u)-\cos (\sqrt{3} u))-\frac{8 \sqrt{2}}{\pi} I_{r}(u),
\end{aligned}
$$

where

$I_{z}(u)=\int_{0}^{\infty} \frac{x^{2} e^{-x^{2} u}}{x^{6}+8} d x, \quad I_{r}(u)=\int_{0}^{\infty} \frac{e^{-x^{2} u}}{x^{6}+8} d x$.

In (4): $\delta_{0}=\gamma_{0}=1, \delta_{n>0}=2, \quad \gamma_{n>0}=(n+1) / 2$; $\phi, \phi_{1}, \vec{e}_{\phi}$ and $r, r_{1}, \vec{e}_{r}$ are the radiated particle offset and the test particle transverse coordinates and corresponding unit orts. The argument $u_{n}$ is equal to $\gamma_{n}^{2 / 3} s / s_{0}$.

The integrands in (6) may be transformed:

$$
\begin{aligned}
& \frac{x^{2}}{x^{6}+8}=\sum_{j=1}^{3} \frac{A_{j}^{1}}{x^{2}+\left(B_{j}\right)^{2}}, \quad \frac{1}{x^{6}+8}=\sum_{j=1}^{3} \frac{A_{j}^{2}}{x^{2}+\left(B_{j}\right)^{2}} \\
& A_{1}^{1}=-2 A_{1}^{2}=-1 / 6, A_{2,3}^{1}=-2 A_{2,3}^{2}=(1 \mp i \sqrt{3}) / 12 \\
& B_{1}=\sqrt{2}, \quad B_{2,3}=(1 \mp i \sqrt{3}) / \sqrt{2} .
\end{aligned}
$$

After integration one obtain:

$$
\begin{aligned}
& f_{z}(s)=\left\{4 e^{-s} \cos (\sqrt{3} s)+\xi(i \sqrt{2 s})-\right. \\
& \left.-\xi\left(e^{i \pi / 6} \sqrt{2 s}\right)-\xi\left(-e^{-i \pi / 6} \sqrt{2 s}\right) / 3\right\} \\
& f_{r}(s)=\left\{2 e^{-s}(\sqrt{3} \sin (\sqrt{3} s)-\cos (\sqrt{3} s))+\xi(i \sqrt{2 s})+\right. \\
& \left.e^{-i \pi / 6} \xi\left(e^{i \pi / 6} \sqrt{2 s}\right)+e^{i \pi / 6} \xi\left(-e^{-i \pi / 6} \sqrt{2 s}\right)\right\} / 3
\end{aligned}
$$

where $\xi(s)=\exp \left(-s^{2}\right) \operatorname{erfc}(-i s)$ is a complex error function [3]. This presentation is equivalent to the obtained in [2], but is a faster calculated and due to its infinity times differentiability may be expanded in series: 


$$
\begin{aligned}
& f_{z}(s)=\sum_{k=0}^{\infty} c_{k} s^{3 k}+\sum_{k=0}^{\infty} d_{k} s^{3 k-3 / 2} \\
& f_{r}(s)=s \sum_{k=0}^{\infty} \tilde{c}_{k} s^{3 k}+s \sum_{k=1}^{\infty} \tilde{d}_{k} s^{3 k-3 / 2} \\
& c_{k}=2^{3 k} /(3 k !), \quad d_{k}=-\sqrt{2 / \pi} 2^{6 k-3} /(6 k-3) ! ! \\
& \tilde{c}_{k}=2^{3 k+1} /(3 k+1) !, \tilde{d}_{k}=-\sqrt{2 / \pi} 2^{6 k-1} /(6 k-1) ! !
\end{aligned}
$$

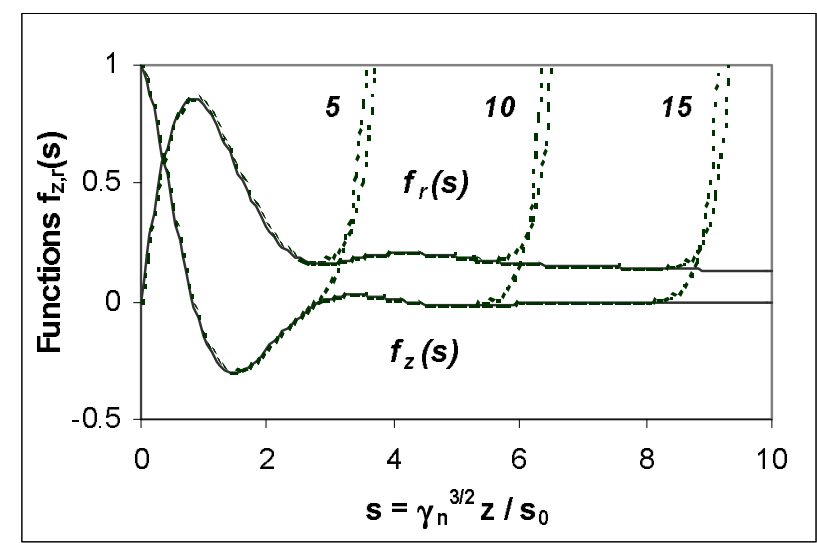

Figure 1. Functions $f_{z}(s)$ and $f_{r}(s)$, calculated exactly (solid) and by 5, 10 and 15 terms of expansion (9) (dashed).

The consecutive terms of series (9) has an alternative signs $\left(d_{k}<0, c_{k}>0, d_{k+1}<0\right)$ and decreases by the module with the number increasing (Laibnitz series) and uniformly converged. In Fig.1 the functions $f_{z, s}(s)$, calculated exactly by help of formulae (8) and by the series (9) interrupted on different steps, are plotted.

\subsection{Gaussian Bunch}

The wake functions for the bunch with the arbitrary charge distribution may be obtained from (4) by help of substitution instead of functions $f_{z}\left(u_{n}\right)$ or $f_{r}\left(u_{n}\right)$ their convolutions with the bunch charge distribution function. Thus, for the Gaussian bunch:

$$
F_{z, r}(z)=(2 \pi)^{-1 / 2} \int_{0}^{\infty} f_{z, r}(\tilde{s} \zeta) e^{-\frac{(\tilde{z}-\tilde{s})^{2}}{2}} d \tilde{s} \text {. }
$$

Here $\tilde{s}_{0}=s_{0} / \gamma_{n}^{3 / 2}, \tilde{z}=z / \sigma_{z}, \tilde{s}=s / \sigma_{z}, \zeta=\sigma_{z} / \tilde{s}_{0}$. The expansion for the comparatively small $\zeta(\zeta \geq 2)$ may be obtained by putting the wake function series (9) into expression (10).

$$
\begin{aligned}
F(z)= & \frac{e^{-z^{2} / 4 \sigma_{z}^{2}}}{\sqrt{2 \pi}} \sum_{k=0}^{\infty}\left\{c_{k} \zeta^{3 k} \Gamma(3 k+1) U\left(3 k+\frac{1}{2},-z / \sigma_{z}\right)+\right. \\
& \left.+d_{k} \zeta^{3 k-3 / 2} \Gamma(3 k-1 / 2) U\left(3 k-1,-z / \sigma_{z}\right)\right),
\end{aligned}
$$

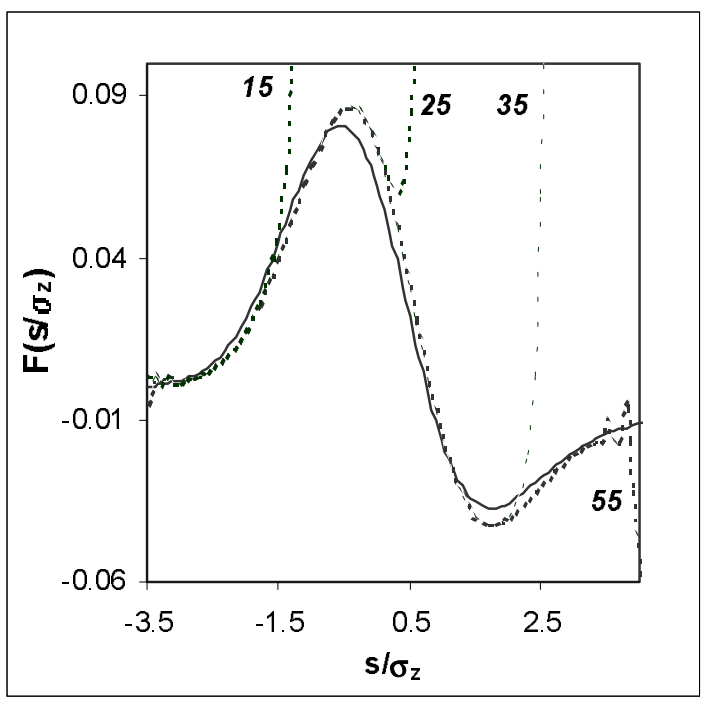

Figure 2. The normalized longitudinal resistive wake function, $\sigma_{z} / s_{0}=2.5$. Dotted curves plotted by help of expansion (11) with different terms of sum. These curves are detached from the different parts of the directly numerical calculated wake potential curve (dot-dashed). Also shown solid curve is calculated by help of the first term of expansion (12). The curves included second, third and forth terms of expansion (12) are coincided (in the presented scale) with the exact calculated wake potential curve (dot-dashed). The difference of these curves is shown in the next figure (Fig.3).

where $U(a, x)$ is a function of parabolic cylinder [3]. The several first terms of this expansion give a good coincidence with the directly integrated expression (10) for the case of comparatively small $\zeta$ (Fig.2). The solution for the arbitrary values of $\tilde{s}_{0} / \sigma$ may be obtained by help of insertion in (10) wake function in the form of (8) and by parts integration. The result is a series expansion by positive degree of $\tilde{s}_{0} / \sigma_{z}$ :

$$
\begin{aligned}
& F(\vec{z})=-\sum_{k=1}^{\infty} \frac{\zeta^{-3 k}}{2^{3 k}}\left\{\zeta^{3 / 2} \frac{d^{3 k-2}}{d \bar{z}^{3 k-2}} Q_{ \pm}(\tilde{z})-\frac{1}{\sqrt{2 \pi}} \frac{d^{3 k-1}}{d \bar{z}^{3 k-1}} e^{-\frac{\tilde{z}^{2}}{2}}\right\}, \\
& Q_{ \pm}(z)=\sqrt{ \pm} z e^{-z^{2} / 4}\left\{I_{-1 / 4}\left(z^{2} / 4\right) \pm I_{1 / 4}\left(z^{2} / 4\right)\right\}
\end{aligned}
$$

with positive sign for $z>0$ and negative one for $z<0$; $I_{ \pm 1 / 4}(z)$ are the modified Bessel functions [3]. The main term of this expansion is equal to obtained by Piwinski [4] low frequency approximation:

$$
\begin{gathered}
\frac{d}{d z} Q_{ \pm}(\tilde{z})=2^{-1}( \pm \tilde{z})^{3 / 2} e^{-u}\left\{-I_{1 / 4}(u)+I_{-3 / 4}(u) \mp\right. \\
\left.\mp I_{-1 / 4}(u) \pm I_{3 / 4}(u)\right\}, \quad u=\tilde{z}^{2} / 4
\end{gathered}
$$

On Fig. 3 is represent the series consistent convergence to the exact calculated with the term number increasing. 


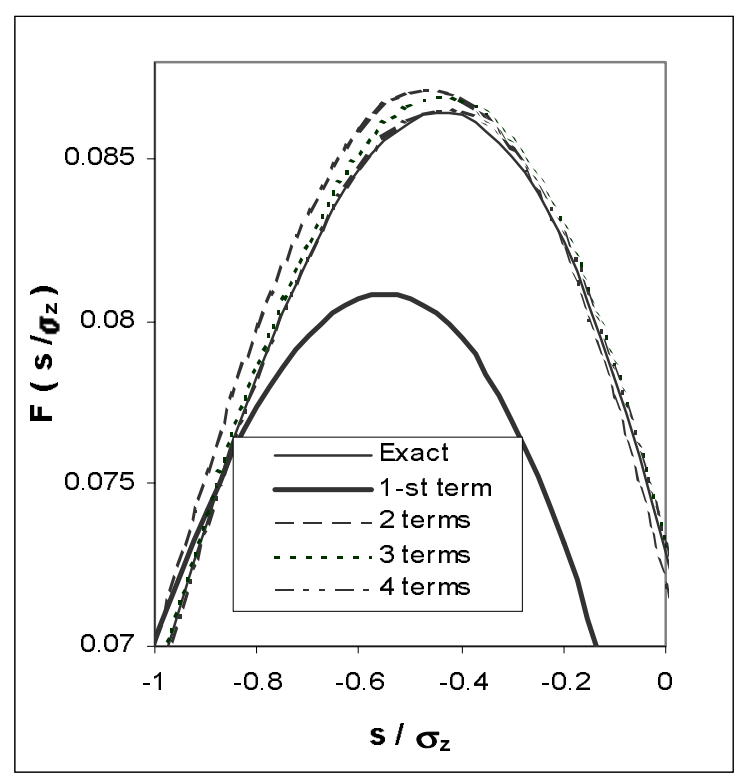

Figure 3. The presentation in detail of approximation (12) convergence to the exact calculated result; $\sigma_{z} / s_{0}=2.5$.

\section{AC CONDUCTIVITY}

In ac case the frequency dependence of conductivity is $\sigma=\sigma_{0} /(1-i \omega \tau)$, where $\sigma_{0}$ is the static conductivity and $\tau$ is the relaxation time of the metal. After changing of designations: $\tilde{\lambda}=-i \lambda / \alpha, \kappa=i \tilde{\lambda}^{2} /\left(2-\tilde{\lambda}^{2} \Gamma\right)$ and $\Gamma=c \tau / s_{0}$, the impedance (1) versus $\tilde{\lambda}$ may be rewrite as follows:

$Z=-\frac{2}{c b s_{0} \alpha} \frac{\tilde{\lambda}\left(2-\tilde{\lambda}^{2} \Gamma\right)}{\left(2-\tilde{\lambda}^{2} \Gamma\right)^{2}+\tilde{\lambda}^{3} / 2}$

with the four roots of denominator:

$\tilde{\lambda}_{1,2}=-1 / 8 \Gamma^{2}-g / 2 \Gamma^{2} \mp e_{-} / 2 \Gamma^{2}$,

$\tilde{\lambda}_{3,4}=-1 / 8 \Gamma^{2}+g / 2 \Gamma^{2} \mp e_{+} / 2 \Gamma^{2}$,

$g=\sqrt{a+b}, \quad a=1 / 16+8 \Gamma^{3} / 3, f=-1 / 8-8 \Gamma^{3}$

$b=3^{-1} \Gamma^{2}\left(64 \Gamma^{2} / d+d\right), e_{\mp}=(2 a-b \mp f / 4 g)^{1 / 2}$

$d=2^{-1 / 3}\left(27+1024 \Gamma^{3}+3 \sqrt{3} \sqrt{27+2048 \Gamma^{3}}\right)^{1 / 3}$.

The first two of roots are real and another two are complex (the term $e_{+}$is imaginary for arbitrary $\Gamma>0$ ). In the de limit $(\Gamma=0): \tilde{\lambda}_{1}=-\infty, \tilde{\lambda}_{2}=-2$ and $\tilde{\lambda}_{3,4}=1 \mp i \sqrt{3}$. Only for two last roots the corresponding values of variable $\kappa: \kappa_{3,4}=i \tilde{\lambda}_{3,4}^{2} \times$ $\times\left(2-\tilde{\lambda}_{3,4}^{2} \Gamma\right)^{-1}$ have negative imaginary parts. The resonance term is the sum of two residues (Fig.4):

$W_{z}^{R}(s)=-\frac{8}{b^{2}} \sum_{j=3,4} \frac{\left(2-\tilde{\lambda}_{j}^{2} \Gamma\right)^{-1} \tilde{\lambda}_{j} e^{-i \kappa_{j} s / s_{0}}}{\left(4 \Gamma^{2} \lambda_{j}^{2}+1.5 \lambda_{j}-8 \Gamma\right)}$.

At $\Gamma \rightarrow 0$ this gives the monopole resonant term of the longitudinal potential for $\mathrm{dc}$ case [2]:

$$
W_{z}^{R}(s)_{\Gamma \rightarrow 0}=-16 / 3 b^{2} e^{-s / s_{0}} \cos \left(\sqrt{3} s / s_{0}\right)
$$

The term, arises from the integration over $\kappa$ along of the both sides of cut from $\kappa=0$ up to $\kappa=-i / \Gamma$ (Fig.4) may be presented as follows:

$$
W_{z}^{0}(s)=\frac{16 \sqrt{2}}{b^{2} \pi} \int_{0}^{\infty} \frac{x^{2}\left(1+x^{2} \Gamma\right)}{8 \cdot\left(1+x^{2} \Gamma\right)^{4}+x^{6}} e^{-\frac{s}{s_{0} 1+x^{2}} \Gamma} d x
$$

and tends to the corresponding non-resonant term of wake function for dc conductivity at $\Gamma \rightarrow 0$.

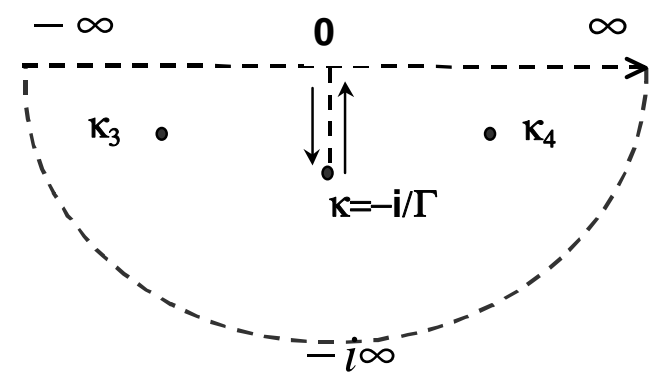

Figure 4. The contour of integration.

\section{CONCLUSION}

Obtained results were used for the TESLA-FEL transport line resistive wakes influence calculation [5].

The results of this report can be useful for wide range of the resistive wake field dominated effects with dc and ac conductivity of the wall material.

\section{REFERENCES}

[1.] A.W. Chao, "Physics of Collective Beam Instabilities in High Energy Accelerators", New York, John Willey \& Sons, Inc., 1993.

[2.] K.L.F. Bane and $M$ Sands,. The Short-Range Resistive Wall Wakefield", SLAC-PUB-95-7074, Dec. 1995.

[3.] M. Abramovits and I.A Stegun., "Handbook of Mathematical Functions", Number 55. Department of Commerce, 2 edition, 1964.

[4.] A Piwinski, "Wake Fields and Ohmic Losses in Round Vacuum Chambers," DESY HERA 92-11, May 1992.

[5.] M.I. Ivanian, V.M.Tsakanov, "Summary of the Resistive Wake-Field Effects in TESLA-FEL Transfer Line”, TESLA-FEL 2000-25, Dec. 2000. 